\title{
Comparison of Optic Nerve Head Parameter Measurements Obtained by Time-domain and Spectral-domain Optical Coherence Tomography
}

\author{
Giacomo Savini, MD, * Piero Barboni, MD, $+\$$ Michele Carbonelli, MD, ${ }^{*}$ Alessandra Sbreglia, OD, $\dagger$ \\ Giulia Deluigi, $M D, \S$ and Vincenzo Parisi, $M D^{*}$
}

\begin{abstract}
Purpose: To compare the measurements of the optic nerve head $(\mathrm{ONH})$ parameters provided by time-domain (TD) and spectraldomain (SD) optical coherence tomography (OCT).

Methods: Four ONH parameters were analyzed: optic disc area, rim area, cup-to-disc area ratio (CDR), and vertical cup-to-disc ratio (VCDR). Stratus OCT and Cirrus HD-OCT were used to obtain measurements by TD-OCT and SD-OCT, respectively. Stratus OCT measurements were collected before and after manual correction of the $\mathrm{ONH}$ edges.
\end{abstract}

Results: Twenty healthy participants and 20 patients with glaucomatous eyes were enrolled. Although manual correction of Stratus OCT measurements reduced the differences compared with Cirrus HD-OCT, the latter measured a smaller mean disc area than Stratus OCT in healthy $\left(2.02 \pm 0.31\right.$ vs. $2.18 \pm 0.29 \mathrm{~mm}^{2}$, $P=0.0003)$ and glaucomatous eyes $(1.92 \pm 0.35$ vs. $2.19 \pm$ $\left.0.38 \mathrm{~mm}^{2}, P<0.0001\right)$. Cirrus HD-OCT measured a smaller rim area than Stratus OCT in healthy $\left(1.31 \pm 0.30\right.$ vs. $1.56 \pm 0.32 \mathrm{~mm}^{2}$, $P<0.0001)$ and glaucomatous eyes $(0.80 \pm 0.25$ vs. $0.97 \pm$ $\left.0.36 \mathrm{~mm}^{2}, P=0.0052\right)$, a higher CDR $(0.55 \pm 0.11$ vs. $0.49 \pm 0.11$, $P<0.0001)$ and VCDR in healthy eyes $(0.55 \pm 0.11$ vs. $0.49 \pm$ $0.11, P<0.0001)$, and a higher $\mathrm{CDR}$ in glaucomatous eyes $(0.74 \pm 0.10$ vs. $0.58 \pm 0.18, P<0.0001)$. No statistically significant differences were detected for VCDR in glaucomatous eyes. All measured values showed good correlation $(r \geq 0.70)$. Large $95 \%$ limits of agreement between the 2 devices were found for most parameters.

Conclusions: $\mathrm{ONH}$ parameter measurements provided by TD-OCT and SD-OCT show significant differences and cannot be considered interchangeable.

Key Words: optic nerve, optical coherence tomography, glaucoma

( J Glaucoma 2013;22:384-389)

$\mathrm{O}$ ptical coherence tomography (OCT) is a noninvasive technology that uses near-infrared light to produce high-resolution cross-sectional images of the retina and the optic nerve. ${ }^{1}$ Over the last 15 years, it has been used extensively to image the posterior pole of the eye in several diseases. Structural changes of the optic disc have been commonly assessed and quantified by measuring the thickness of the peripapillary retinal nerve fiber layer

Received for publication November 28, 2010; accepted January 9, 2012. From the *G.B. Bietti Eye Foundation-IRCCS, Rome; †Studio

Oculistico d'Azeglio; \$Department of Neurological Sciences, University of Bologna; and §Private practice, Bologna, Italy.

Disclosure: The authors declare no conflict of interest.

Reprints: Giacomo Savini, MD, G.B. Bietti Eye Foundation-IRCCS,

Via Livenza 3, Rome, Italy 00198 (e-mail: giacomo.savini@alice.it). Copyright (C) 2013 by Lippincott Williams \& Wilkins

DOI:10.1097/IJG.0b013e31824c9423
$(\mathrm{RNFL}){ }^{2-6}$ whereas optic nerve head $(\mathrm{ONH})$ analysis by OCT received less attention until a few years ago. However, it has finally gained importance for at least 3 reasons: (1) it improves the ability to discriminate between healthy and glaucomatous eyes ${ }^{7-11}$; (2) $\mathrm{ONH}$ parameters play an important role in other diseases, such as Leber hereditary optic neuropathy, dominant optic atrophy, and nonarteritic ischemic optic neuropathy ${ }^{12-14}$; (3) the ONH size influences the RNFL thickness measurements and, as a consequence, the sensitivity of Stratus OCT with respect to glaucoma detection, as a decrease in sensitivity has been reported in subjects with larger discs. ${ }^{15-17}$ The latter studies were carried out by means of the Stratus OCT (Carl Zeiss Meditec, Dublin, CA), which uses time-domain (TD) OCT. The most recent development in OCT imaging uses spectral-domain (SD) technology, which improves data acquisition due to a higher scanning speed and resolution. ${ }^{18}$ Cirrus HD-OCT (Carl Zeiss Meditec) is one of the most widely used devices with SD-OCT technology. Recent studies have shown that RNFL thickness measurements by this instrument are not interchangeable with those obtained by TD-OCT. ${ }^{19,20}$ No comparisons are yet available for the $\mathrm{ONH}$ analysis protocol, which was released on Cirrus HD-OCT in 2010 and has already been shown to provide repeatable measurements with a diagnostic accuracy as high as that achieved for RNFL thickness. ${ }^{21-23}$

Given that glaucoma is a progressive disease, and so longitudinal follow-up is crucial, and that several thousands of glaucomatous patients who have been scanned with Stratus OCT since 2002 (the year it was made commercially available) will now be scanned with Cirrus HD-OCT, we were interested in assessing the level of agreement between the 2 instruments in measuring $\mathrm{ONH}$ parameters.

\section{METHODS}

For this prospective study, all participants were enrolled from a private practice (Studio Oculistico d'Azeglio, Bologna, Italy). Before being included in the study, each subject was informed of its purpose and gave a written consent to participate. The study protocol had been approved by the Institutional Review Board of the Department of Neurological Sciences of the University of Bologna, Italy. Study methods adhered to the provisions of the Declaration of Helsinki guidelines for research involving human participants.

All subjects were whites and were classified as healthy or glaucomatous. Healthy subjects were recruited among volunteers from the staff and patients with minor refractive disorders examined in the above-mentioned private practice. Inclusion criteria for healthy subjects were as follows: 
a best corrected visual acuity $>20 / 25$, refractive error between -3 and $+3 \mathrm{D}$ of sphere or between -2 and $+2 \mathrm{D}$ of cylinder, normal intraocular pressure $<21 \mathrm{~mm} \mathrm{Hg}$, normal appearance of the optic disc, no significant ocular disease found by routine ophthalmological examination, no history of glaucoma in the family and/or systemic diseases with possible ocular involvement, such as diabetes mellitus. Glaucomatous patients were recruited among patients routinely followed up at the same private practice. Glaucoma was defined on the basis of the presence of visual field defects (described below) regardless of the $\mathrm{ONH}$ appearance and intraocular pressure. ${ }^{23}$ Peripapillary atrophy (PPA) was not considered as an exclusion criterion (nor was it in healthy eyes).

\section{Visual Field Examination}

Visual field testing was performed using static automated white-on-white threshold perimetry (SITA Standard 24-2, Humphrey Field Analyzer II; Carl Zeiss Meditec). A visual field was defined as reliable when fixation losses, and false-positive and false-negative errors, were $<20 \%$. A visual field defect was defined as glaucomatous when having $\geq 3$ significant $(P<0.05)$ nonedge contiguous points with $\geq 1$ at the $P<0.01$ level on the same side of the horizontal meridian in the pattern deviation plot and had been confirmed with $\geq 2$ consecutive examinations. ${ }^{24}$ Visual field testing had to be performed within 1 month of testing with OCT.

\section{Cirrus HD-OCT ONH Imaging}

All scans were acquired by the same operator with the same Cirrus HD-OCT (software version 5.0) device using the optic disc cube $200 \times 200$ protocol in eyes dilated with tropicamide $1 \%$. The Optic Disc Cube $200 \times 200$ protocol is designed to position the cube scan on the $\mathrm{ONH}$ and to be used primarily for glaucoma analysis. After the patient had been properly seated and aligned, the iris was brought into view using the mouse-driven alignment system, and the ophthalmoscopic image was focused. The ONH was then centered on the live image before the centering and enhancement was optimized. After the scanning process was launched, the instrument's $840-\mathrm{nm}$ wavelength laser beam generated a cube of data measuring $6 \mathrm{~mm} \times 6 \mathrm{~mm}$ after scanning a series of 200 B-scans with 200 A-scans per B-scan (40,000 points).

Only scans with a signal strength $\geq 6$ and without eye movements or blinking artifacts were used for analysis. With regard to $\mathrm{ONH}$ parameters, the algorithm identifies the termination of Bruch membrane as the disc edge. The rim width around the entire circumference of the optic disc is then determined by measuring the thickness of the neuroretinal tissue in the optic nerve as it turns to exit through the opening in Bruch membrane. The following $\mathrm{ONH}$ parameters were analyzed: disc area, rim area, cup-to-disc area ratio (CDR: ratio of cup area to disc area), and vertical cup-to-disc ratio (VCDR: ratio of the vertical line through the cup center to the same vertical line extending to the disc margin). These ONH parameters were automatically generated by a Carl Zeiss Meditec analysis algorithm that does not involve user interaction. ${ }^{21}$

\section{Stratus OCT ONH Imaging}

A second operator performed all OCT measurements with Stratus OCT (software version 4.0) a few minutes after the Cirrus HD-OCT measurements. ONH evaluation consisted of 6 radial scans centered on the ONH and spaced 30 degrees apart (Fast Optic Disc acquisition protocol). Each radial scan included 128 points. The machine automatically defined the edge of the optic disc as the end of the retinal pigment epithelium (RPE)/choriocapillaris layer and used smoothing with fit to circle to fill the gaps between scans. The resultant image could be manually corrected when the machine did not identify the edge correctly (in this case the operator moved one or both cursors on the edge of the RPE/choriocapillaris layer). The optic cup is defined by a cup-offset that by default runs parallel and $150 \mu \mathrm{m}$ anterior to the reference plane as defined by the edge of the RPE. The optic cup is represented by the region below the cup-offset, and the neuroretinal rim by the area above it. Among the several ONH parameters provided by the Stratus OCT software, we analyzed the optic disc area, the rim area, the CDR, and the VCDR. In the present study, we recorded these values before and, when necessary, after the operator had manually corrected the disc edges. In addition, each scan was carefully evaluated to rule out possible artifacts (such as those deriving, eg, from posterior vitreous detachment).

\section{Statistics}

All statistical analyses were performed using GraphPad InStat version 3a for Macintosh (GraphPad Software, San Diego, CA www.graphpad.com). For statistical evaluations, only 1 eye, randomly chosen, was considered for each patient if both eyes were eligible for the study.

Mean values given by Cirrus HD and Stratus OCT were compared using a paired $t$ test. Correlation with Pearson $r$ coefficient (for parametric data) and Spearman $r$ coefficient (for nonparametric data) was performed to assess how consistently the measurements of the 2 instruments varied together.

Agreement between Cirrus HD-OCT and Stratus OCT measurements was evaluated according to the method described by Bland and Altman. ${ }^{25,26}$ Bland and Altman plots allow us to investigate the existence of any systematic difference between measurements (ie, fixed bias). The mean difference is the estimated bias, and the SD of the differences measures the random fluctuations around this mean. The 95\% limits of agreement (LoA) were defined as means \pm 2 SD of the differences between the 2 measurement techniques.

Bland and Altman plots were also used to investigate the existence of proportional bias, which would indicate that the methods do not agree equally across the range of measurements (ie, the LoA will depend on the actual measurement). To evaluate this relationship formally, the difference between the methods was regressed on the average of the 2 methods.

\section{RESULTS}

Twenty healthy subjects (mean age: $54.6 \pm 19.2 \mathrm{y}$ ) and 20 glaucomatous patients (mean age: $59.3 \pm 18.7 \mathrm{y}$ ) were enrolled (none had to be excluded because of the abovementioned criteria or because the signal strength was $<6$ ); PPA was detected in $5(25 \%)$ of the healthy and $9(45 \%)$ of the glaucomatous eyes. Among patients, 16 had been diagnosed with open-angle glaucoma and 4 with normaltension glaucoma. The visual field mean defect of glaucomatous eyes was $-6.78 \pm 5.22 \mathrm{~dB}$. No artifacts in the automatic ONH analysis protocol by Cirrus HD-OCT were detected after careful checking of each examination. With regard to Stratus OCT scans, manual correction of the 


\begin{tabular}{|c|c|c|c|c|c|}
\hline & Cirrus HD-OCT & Stratus OCT (Automatic) & $P^{*}$ & Stratus OCT (Manual) & $\boldsymbol{P} \dagger$ \\
\hline \multicolumn{6}{|l|}{ Healthy eyes } \\
\hline Optic disc area $\left(\mathrm{mm}^{2}\right)$ & $2.02 \pm 0.31$ & $2.29 \pm 0.40$ & $<0.0001$ & $2.18 \pm 0.29$ & 0.0003 \\
\hline Rim area $\left(\mathrm{mm}^{2}\right)$ & $1.31 \pm 0.30$ & $1.60 \pm 0.37$ & $<0.0001$ & $1.56 \pm 0.32$ & $<0.0001$ \\
\hline Cup-to-disc area ratio & $0.55 \pm 0.16$ & $0.31 \pm 0.13$ & $<0.0001$ & $0.32 \pm 0.15$ & $<0.0001$ \\
\hline Vertical cup-to-disc ratio & $0.55 \pm 0.11$ & $0.50 \pm 0.17$ & 0.0053 & $0.49 \pm 0.11$ & 0.0004 \\
\hline \multicolumn{6}{|l|}{ Glaucoma } \\
\hline Optic disc area $\left(\mathrm{mm}^{2}\right)$ & $1.92 \pm 0.35$ & $2.39 \pm 0.33$ & $<0.0001$ & $2.19 \pm 0.38$ & $<0.0001$ \\
\hline Rim area $\left(\mathrm{mm}^{2}\right)$ & $0.80 \pm 0.25$ & $1.02 \pm 0.33$ & 0.0007 & $0.97 \pm 0.36$ & 0.0052 \\
\hline Cup-to-disc area ratio & $0.74 \pm 0.10$ & $0.56 \pm 0.16$ & $<0.0001$ & $0.58 \pm 0.18$ & $<0.0001$ \\
\hline Vertical cup-to-disc ratio & $0.73 \pm 0.11$ & $0.72 \pm 0.11$ & NS $(0.85)$ & $0.72 \pm 0.11$ & NS $(0.74)$ \\
\hline
\end{tabular}

*Comparison between Cirrus HD-OCT and Stratus OCT automatic measurements.

†Comparison between Cirrus HD-OCT and Stratus OCT measurements after manual correction.

NS indicates not significant.

ONH edges was carried out for 19 of the 20 healthy and 19 of the 20 glaucomatous eyes (in the only case that did not require it, the same values were entered in both automatic and manual measurements for statistical analysis).

\section{Disc and Rim Areas}

Cirrus HD-OCT measured smaller disc and rim areas than Stratus OCT in both healthy subjects and glaucoma patients; the difference slightly decreased after manual correction of the $\mathrm{ONH}$ edges identified by Stratus OCT (Table 1). As expected, disc and rim area measurements, as performed by the 2 devices, showed good correlation (Table 2 and Fig. 1). The correlation coefficient was slightly higher with manual than with automatic Stratus OCT measurements.

The difference in disc area (Cirrus HD-OCT minus Stratus OCT manually corrected) was correlated to the difference in rim area for both healthy $\left(P=0.0004, r^{2}=0.51\right)$ and glaucomatous eyes $\left(P=0.0208, r^{2}=0.26\right)$. In both groups, no statistically significant relationship was observed between the difference in disc area and other ONH parameters.

In healthy eyes, Bland-Altman plots for disc and rim area measurements revealed a weak agreement between Cirrus HD-OCT and Stratus OCT (Table 3); manually corrected measurements by Stratus OCT achieved slightly narrower $95 \%$ LoA than the automatic ones. A proportional bias was detected for the disc area, as the difference between Cirrus HD-OCT and Stratus OCT automatic measurements became higher with increasing disc size $\left(r=-0.45, r^{2}=0.20, P=0.046\right)$. Such a bias was not observed for manual disc area measurements obtained with Stratus OCT, or for rim area measurements (with or without manual correction of Stratus OCT values).

In the case of glaucomatous eyes, the 95\% LoA for both disc area (Fig. 2) and rim area were even larger than in the controls, with slightly better results again for manually corrected measurements by Stratus OCT. No proportional biases for disc and rim area measurements were detected in case of glaucoma.

\section{CDR and VCDR}

In both healthy subjects and glaucoma patients, Cirrus HD-OCT provided higher CDR measurements than Stratus OCT (although the difference between Cirrus HD-OCT and Stratus OCT manual measurements was not statistically significant in case of glaucoma; Table 1). As far as the VCDR is concerned, Cirrus HD-OCT measured higher values than Stratus OCT in healthy eyes; in contrast, the mean values were quite similar in glaucomatous eyes, where no statistically significant differences between the 2 instruments were detected (Table 1). In both groups, correlation disclosed a strong relationship between CDR and VCDR measurements as performed by the 2 devices (Table 2).

Bland-Altman plots revealed a poor agreement between Cirrus HD-OCT and Stratus OCT CDR measurements in both healthy and glaucomatous eyes (Table 3). The only

TABLE 2. Correlation Coefficients Between Cirrus HD-OCT and Stratus OCT Measurements

\begin{tabular}{|c|c|c|c|c|c|c|}
\hline & \multicolumn{3}{|c|}{$\begin{array}{c}\text { Correlation Between Cirrus HD-OCT and Stratus } \\
\text { OCT Automatic Measurements }\end{array}$} & \multicolumn{3}{|c|}{$\begin{array}{c}\text { Correlation Between Cirrus HD-OCT and Stratus } \\
\text { OCT Manual Measurements }\end{array}$} \\
\hline & $\mathbf{r}$ & $r^{2}$ & $\boldsymbol{P}$ & $\boldsymbol{r}$ & $r^{2}$ & $\boldsymbol{P}$ \\
\hline \multicolumn{7}{|l|}{ Healthy eyes } \\
\hline Optic disc area & 0.86 & 0.74 & $<0.0001$ & 0.87 & 0.76 & $<0.0001$ \\
\hline Rim area & 0.82 & 0.68 & $<0.0001$ & 0.88 & 0.77 & $<0.0001$ \\
\hline Cup-to-disc area ratio & 0.90 & 0.82 & $<0.0001$ & 0.70 & 0.50 & 0.0007 \\
\hline Vertical C/D ratio & 0.81 & 0.66 & $<0.0001$ & 0.86 & 0.74 & $<0.0001$ \\
\hline \multicolumn{7}{|l|}{ Glaucoma } \\
\hline Optic disc area & 0.68 & 0.46 & 0.0009 & 0.79 & 0.63 & $<0.0001$ \\
\hline Rim area & 0.69 & 0.48 & 0.0007 & 0.74 & 0.54 & 0.0002 \\
\hline Cup-to-disc area ratio & 0.89 & 0.79 & $<0.0001$ & $0.77 *$ & 0.59 & $<0.0001$ \\
\hline Vertical C/D ratio & 0.78 & 0.61 & $<0.0001$ & 0.83 & 0.69 & $<0.0001$ \\
\hline
\end{tabular}

Pearson coefficient used for all correlations except those marked with *, showing that Spearman coefficient was used.

NA indicates not available. 


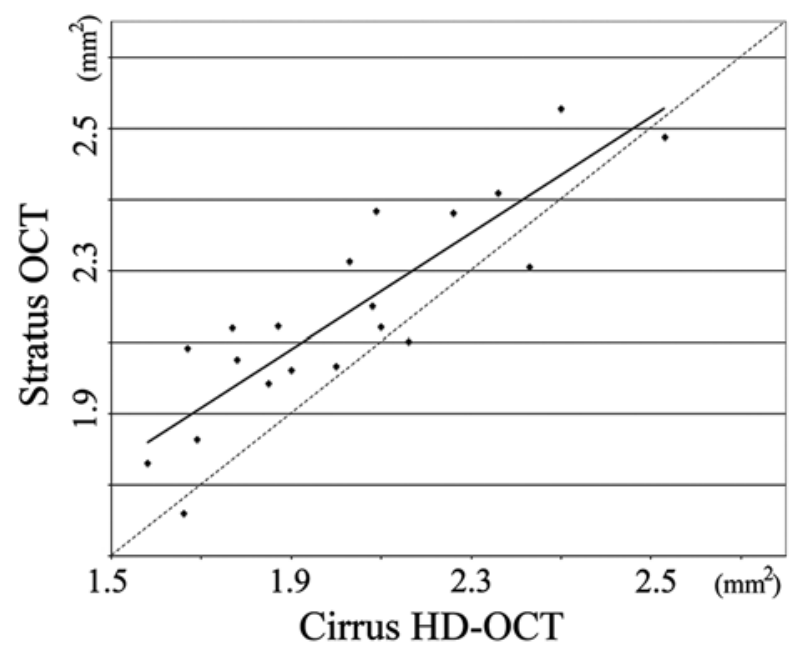

FIGURE 1. A scatter plot of the disc area measurements of healthy eyes. The disc area measured by Cirrus HD-OCT is plotted against the disc area measured by Stratus OCT (with manual correction). The linear regression line (solid) equation is $y=0.8157 x+0.529$ and is compared with the $y=x$ (dashed) line. The Pearson correlation coefficient is 0.87 .

parameter for which narrow 95\% LoA were calculated was the VCDR, especially in glaucomatous eyes. No proportional biases were present for CDR and VCDR.

\section{DISCUSSION}

The results of the current study indicate that most $\mathrm{ONH}$ parameters measured by Cirrus HD-OCT are not interchangeable with those obtained by Stratus OCT because of statistically significant differences in the mean values and poor agreement in both healthy and glaucomatous eyes.

The strongest differences were detected in measurements of the disc and rim areas, for which Cirrus HD-OCT provided lower mean values than Stratus OCT. The reason for such discrepancies is unclear. We can rule out that these results depend on a different camera magnification of the fundus image, as this value is the same for the 2 instruments. ${ }^{27}$ Hence, we can postulate that they are related to the different algorithms used to identify the ONH edges, and more specifically to 2 factors: first, in Cirrus HD-OCT, the algorithm identifies the termination of Bruch membrane as the disc edge, whereas in Stratus OCT, the termination of the RPE/choriocapillaris layer is used to define the disc edge. Second, while the Stratus OCT reconstructs the $\mathrm{ONH}$ shape by interpolating the data of 6 radial scans centered on the $\mathrm{ONH}$ and spaced 30 degrees apart and uses smoothing with fit to circle to fill the gaps between scans (Fig. 3), the Cirrus HD-OCT takes advantage of the higher scanning speed of SD-OCT and analyzes the cube of data of the peripapillary area, with minimal interpolation (although inaccuracies may arise due to the fact that large retinal vessels cause a shadow that makes Bruch membrane opening not any longer visible). Differences are also present in the identification of the rim-cup boundary, but in this case, the Cirrus HD-OCT method has not been revealed by the manufacturer. The difference in disc area values between the 2 instruments was correlated to the difference in rim area, that is, when the disc area measured by Cirrus HDOCT was lower, the rim area measured by the same instrument was lower too. Therefore, we suppose that the difference in rim area can be largely explained by the difference in disc area. In contrast, the difference in disc area does not seem to be related to the difference in the cup-to-disc ratio.

We were not surprised to observe a lower difference and narrower 95\% LoA between Cirrus HD-OCT and Stratus OCT measurements when the latter were manually corrected; although most studies have relied on the automatic algorithm of Stratus OCT to avoid introducing a subjective component into their analysis, ${ }^{11,28}$ other authors have reported that manual correction of the ONH analysis by Stratus OCT leads to a more realistic and more reproducible approach, in view of frequent failed disc margin recognition. ${ }^{29,30}$ Accordingly, in a previous investigation, our own group had to perform manual correction in 78 of the 79 eyes $(98.7 \%$ ) because of imperfect identification of the ONH edges by the Stratus OCT internal software. ${ }^{31}$

Agreement between the 2 instruments in disc and rim area measurements was better for healthy eyes than for glaucomatous eyes. One possible explanation is that the higher incidence of PPA in glaucomatous eyes in our sample might have influenced disc edge detection by one or both instruments.

As expected, the values provided by the 2 devices showed good correlation both in healthy and in glaucomatous eyes (with correlation coefficients higher, again, after manual correction of the Stratus OCT measurements). Consequently, we can speculate that findings from previous

TABLE 3. Ninety-five Percent LoA Between Cirrus HD-OCT and Stratus OCT Measurements of the Optic Nerve Head Parameters

\begin{tabular}{|c|c|c|c|c|}
\hline & \multicolumn{2}{|c|}{$\begin{array}{l}\text { Agreement Between Cirrus HD-OCT and Stratus } \\
\text { OCT Automatic Measurements }\end{array}$} & \multicolumn{2}{|c|}{$\begin{array}{l}\text { Agreement Between Cirrus HD-OCT and Stratus } \\
\text { OCT Manual Measurements }\end{array}$} \\
\hline & Mean Difference (Cirrus - Stratus) & 95\% LoA & Mean Difference (Cirrus - Stratus) & 95\% LoA \\
\hline \multicolumn{5}{|l|}{ Healthy eyes } \\
\hline Disc area $\left(\mathrm{mm}^{2}\right)$ & $-0.27 \pm 0.21$ & -0.68 to +0.15 & $-0.16 \pm 0.16$ & -0.47 to +0.15 \\
\hline Rim area $\left(\mathrm{mm}^{2}\right)$ & $-0.29 \pm 0.21$ & -0.71 to +0.13 & $-0.25 \pm 0.16$ & -0.56 to +0.06 \\
\hline Cup-to-disc area ratio & $0.23 \pm 0.16$ & -0.09 to 0.55 & $0.22 \pm 0.17$ & -0.12 to 0.56 \\
\hline Vertical cup-to-disc ratio & $0.05 \pm 0.07$ & -0.09 to 0.19 & $0.06 \pm 0.06$ & -0.06 to 0.17 \\
\hline \multicolumn{5}{|l|}{ Glaucoma } \\
\hline Disc area $\left(\mathrm{mm}^{2}\right)$ & $-0.46 \pm 0.27$ & -1.01 to +0.08 & $-0.27 \pm 0.24$ & -0.74 to +0.20 \\
\hline Rim area $\left(\mathrm{mm}^{2}\right)$ & $-0.22 \pm 0.24$ & -0.70 to +0.27 & $-0.17 \pm 0.24$ & -0.65 to +0.31 \\
\hline Cup-to-disc area ratio & $0.18 \pm 0.09$ & 0.00 to 0.35 & $0.16 \pm 0.13$ & -0.10 to 0.41 \\
\hline Vertical cup-to-disc ratio & $0.01 \pm 0.07$ & -0.14 to 0.15 & $0.01 \pm 0.06$ & -0.12 to 0.14 \\
\hline
\end{tabular}

LoA indicates limits of agreement. 

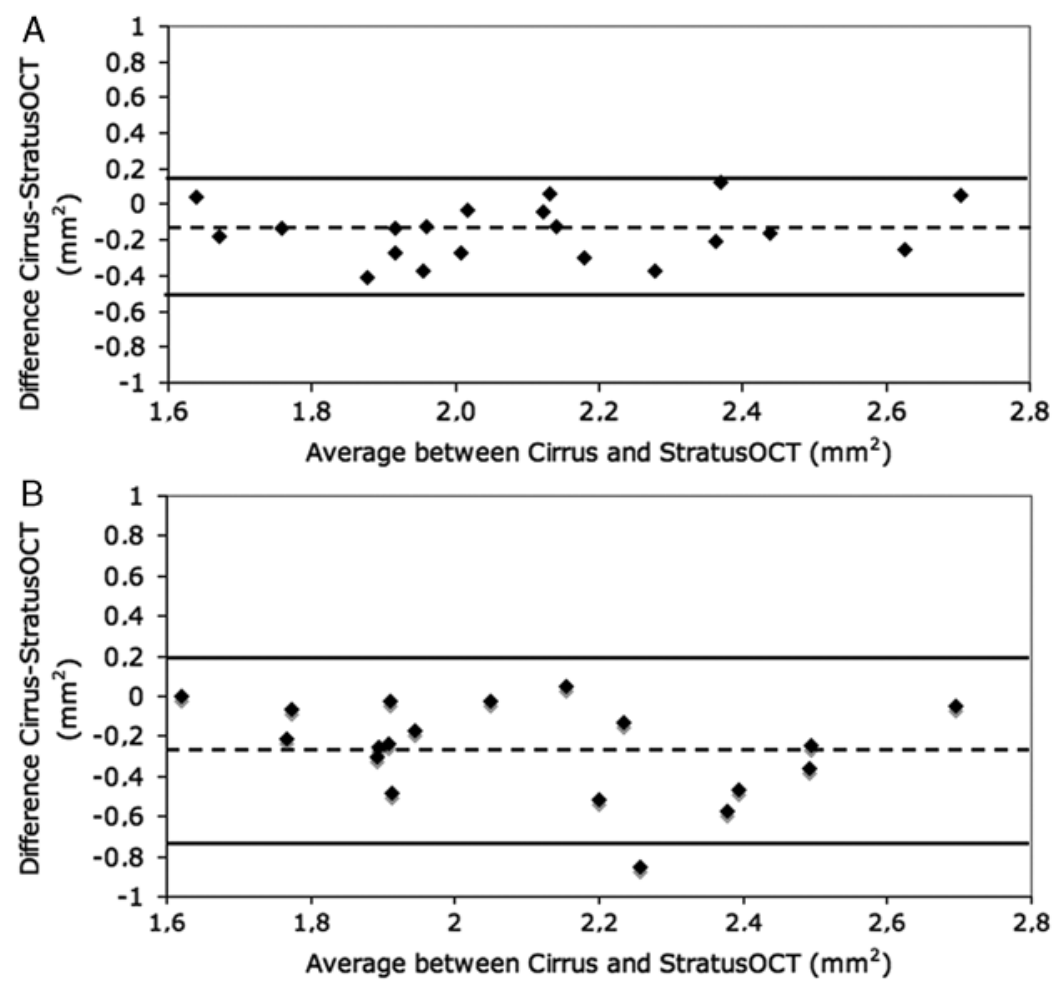

FIGURE 2. Bland-Altman plots showing agreement between Cirrus HD-OCT and manual Stratus OCT measurements of the optic disc area in healthy (A) and glaucomatous eyes (B). Ninety-five percent limits of agreement are larger in glaucomatous (from - 0.74 to $+0.20 \mathrm{~mm}^{2}$ ) than in healthy eyes (from -0.47 to $+0.15 \mathrm{~mm}^{2}$ ).

studies carried out with TD-OCT (eg, the association between large optic discs and better prognosis in Leber hereditary optic neuropathy or the association between small optic discs and dominant optic atrophy with OPA1 gene mutations) ${ }^{13,32}$ are also likely to prove valid once measurements are performed using Cirrus HD-OCT. Further studies are under way to confirm this hypothesis.
Agreement between the 2 instruments was also poor for CDR measurements in both groups of subjects. In healthy eyes, the agreement was poor for VCDR also, whereas in glaucomatous eyes, the latter parameter showed a small difference between the 2 devices and narrow 95\% LoA. The reason why VCDR values in glaucoma were the only measurements with good agreement between the 2 instruments is

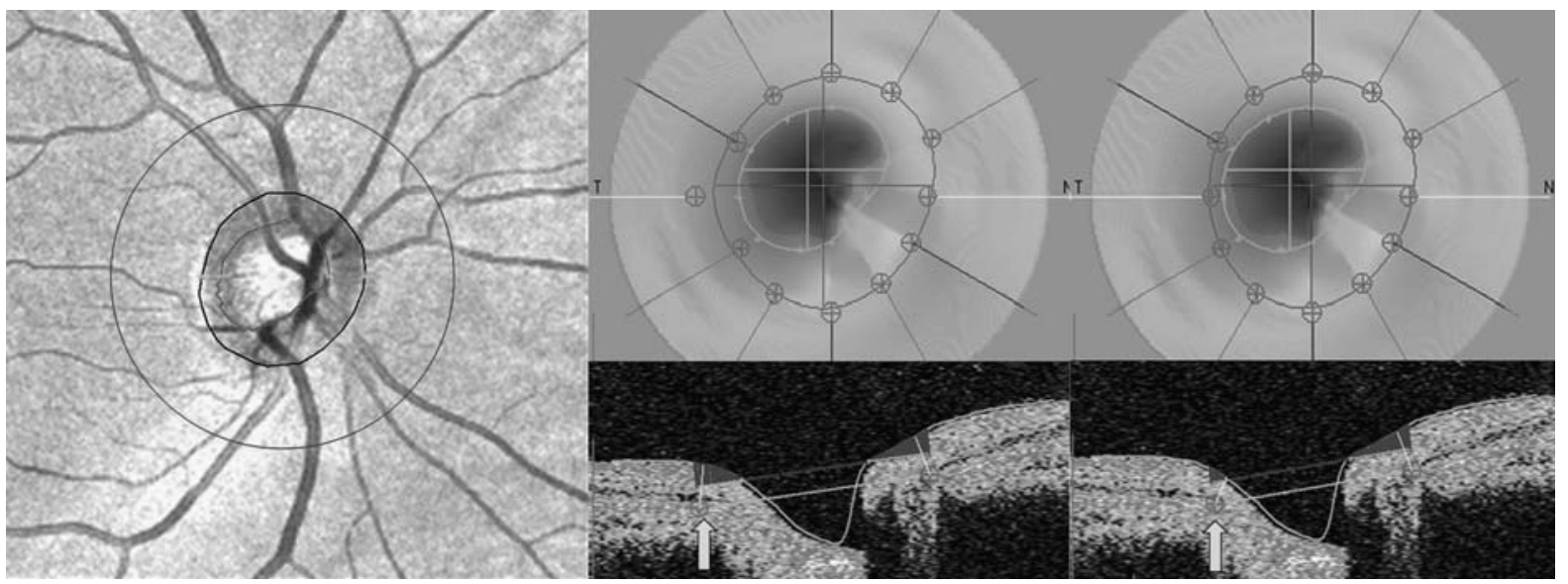

FIGURE 3. Left: optic nerve head identification by Cirrus HD-OCT. Middle: Automatic identification of optic nerve head by Stratus OCT does not correctly detect the temporal edge of the disc (top) due to inappropriate detection of the retinal pigment epithelium (RPE) termination (bottom, white arrow). Right: manual adjustment allows correct identification of the disc edge (top) due to the right position of the RPE termination (bottom, white arrow). The Stratus OCT reconstructs the shape of the optic nerve head by interpolating the data of 6 radial scans and uses smoothing with fit to circle to fill the gaps between scans. 
not clear. However, we believe that automatic detection of the cup-rim boundary is simpler in glaucomatous eyes than in healthy eyes, due to the steeper slope of the ONH cup in eyes with glaucomatous optic atrophy. ${ }^{33,34}$

This study has some limitations that warrant further investigation. First, glaucomatous patients were not classified on the basis of the severity of visual field defect, using grading scales such as the one described by Hodapp et al. ${ }^{35}$ Second, we did not aim to compare the diagnostic accuracy (sensitivity and specificity) of the 2 instruments for glaucoma detection. Given that the diagnostic accuracy of ONH measurements by TD-OCT has not yet been shown to be superior to RNFL thickness measurements, it will be interesting to investigate this issue by means of SD-OCT. Finally, we did not adjust the cup-offset of the Stratus OCT, as suggested previously by our own group. ${ }^{31}$

In conclusion, our data show that $\mathrm{ONH}$ measurements provided by Cirrus HD-OCT and Stratus OCT are highly correlated but significantly different (the only exception being VCDR in glaucomatous eyes) and thus cannot be used interchangeably.

\section{REFERENCES}

1. Huang D, Swanson EA, Lin CP, et al. Optical coherence tomography. Science. 1991;254:1178-1181.

2. Schuman JS, Pedut-Kloizman T, Hertzmark E, et al. Reproducibility of nerve fiber layer thickness measurements using optical coherence tomography. Ophthalmology. 1996;103:1889-1898.

3. Pieroth L, Schuman JS, Hertzmark E, et al. Evaluation of focal defects of the nerve fiber layer using optical coherence tomography. Ophthalmology. 1999;106:570-579.

4. Bowd C, Zangwill LM, Berry CC, et al. Detecting early glaucoma by assessment of retinal nerve fiber layer thickness and visual function. Invest Ophthalmol Vis Sci. 2001;42:1993-2003.

5. Bagga H, Greenfield DS. Quantitative assessment of structural damage in eyes with localized visual field abnormalities. Am J Ophthalmol. 2004;137:797-805.

6. Medeiros FA, Zangwill LM, Bowd C, et al. Comparison of the GDx VCC scanning laser polarimeter, HRT II confocal scanning laser ophthalmoscope, and stratus OCT optical coherence tomograph for the detection of glaucoma. Arch Ophthalmol. 2004;122:827-837.

7. Huang ML, Chen HY, Lin JC. Rule extraction for glaucoma detection with summary data from StratusOCT. Invest Ophthalmol Vis Sci. 2007;48:244-250.

8. Manassakorn A, Nouri-Mahdavi K, Caprioli J. Comparison of retinal nerve fiber layer thickness and optic disk algorithms with optical coherence tomography to detect glaucoma. Am J Ophthalmol. 2006;141:105-115.

9. Naithani P, Sihota R, Sony P, et al. Evaluation of optical coherence tomography and Heidelberg retinal tomography parameters in detecting early and moderate glaucoma. Invest Ophthalmol Vis Sci. 2007;48:3138-3145.

10. Leung CK, Chan W, Hui Y, et al. Analysis of retinal nerve fiber layer and optic nerve head in glaucoma with different reference plane offsets, using optical coherence tomography. Invest Ophthalmol Vis Sci. 2005;46:891-899.

11. Medeiros FA, Zangwill LM, Bowd C, et al. Evaluation of retinal nerve fiber layer, optic nerve head, and macular thickness measurements for glaucoma detection using optical coherence tomography. Am J Ophthalmol. 2005;139:44-55.

12. Ramos Cdo V, Bellusci C, Savini G, et al. Association of optic disc size with development and prognosis of Leber's hereditary optic neuropathy. Invest Ophthalmol Vis Sci. 2009;50:1666-1674.

13. Barboni P, Carbonelli M, Savini G, et al. OPA1 mutations associated with dominant optic atrophy influence optic nerve head size. Ophthalmology. 2010;117:1547-1553.

14. Chan CKM, Cheng ACO, Leung CKS, et al. Quantitative assessment of optic nerve head morphology and retinal nerve fibre layer in non-arteritic anterior ischaemic optic neuropathy with optical coherence tomography and confocal scanning laser ophthalmoloscopy. Br J Ophthalmol. 2009;93:731-735.

15. Savini G, Zanini M, Carelli V, et al. Correlation between retinal nerve fibre layer thickness and optic nerve head size: an optical coherence tomography study. Br J Ophthalmol. 2005;89:489-492.

16. Budenz DL, Anderson DR, Varma R, et al. Determinants of normal retinal nerve fiber layer thickness measured by Stratus OCT. Ophthalmology. 2007;114:1046-1052.

17. Medeiros FA, Zangwill LM, Bowd C, et al. Influence of disease severity and optic disc size on the diagnostic performance of imaging instruments in glaucoma. Invest Ophthalmol Vis Sci. 2006;47:1008-1015.

18. Wojtkowski M, Leitgeb R, Kowalczyk A, et al. In vivo human retinal imaging by Fourier-domain optical coherence tomography. J Biomed Opt. 2002;7:457-463.

19. Knight OR, Chang RT, Feuer WJ, et al. Comparison of retinal nerve fiber layer measurements using time domain and spectral domain optical coherence tomography. Ophthalmology. 2009; 116:1271-1277.

20. Moreno-Montañés J, Olmo N, Alvarez A, et al. Cirrus highdefinition optical coherence tomography compared with Stratus optical coherence tomography in glaucoma diagnosis. Invest Ophthalmol Vis Sci. 2010;51:335-343.

21. Mwanza JC, Chang RT, Budenz DL, et al. Reproducibility of peripapillary retinal nerve fiber layer thickness and optic nerve head parameters measured with Cirrus ${ }^{\mathrm{TM}}$ HD-OCT in glaucomatous eyes. Invest Ophthalmol Vis Sci. 2010;51:5724-5730.

22. Savini G, Carbonelli M, Parisi V, et al. Repeatability of optic nerve head parameters measured by spectral-domain OCT in healthy eyes. Ophthalmic Surg Lasers Imaging. (In press).

23. Mwanza JC, Oakley JD, Budenz DL, et al. Ability of Cirrus HD-OCT optic nerve head parameters to discriminate normal from glaucomatous eyes. Ophthalmology. 2011;118:241-248.

24. Leung CK, Choi N, Weinreb RN, et al. Retinal nerve fiber layer imaging with spectral-domain optical coherence tomography: pattern of RNFL defects in glaucoma. Ophthalmology. (In press).

25. Bland JM, Altman DG. Statistical methods for assessing agreement between two methods of clinical measurement. Lancet. 1986;1:307-310.

26. Altman DG, Bland JM. Measurement in medicine: the analysis of method comparison studies. Statistician. 1983;32:307-317.

27. Kang SH, Hong SW, Im SK, et al. Effect of myopia on the thickness of the retinal nerve fiber layer measured by Cirrus HD optical coherence tomography. Invest Ophthalmol Vis Sci. 2010;51:4075-4083

28. Arnalich-Montiel F, Muñoz-Negrete FJ, Rebolleda G, et al. Cup-to-disc ratio: agreement between slit-lamp indirect ophthalmoscopic estimation and stratus optical coherence tomography measurement. Eye. 2007;21:1041-1049.

29. Iliev ME, Meyenberg A, Garweg JG. Morphometric assessment of normal, suspect and glaucomatous optic discs with StratusOCT and HRTII. Eye. 2006;20:1288-1299.

30. Kamppeter BA, Schubert KV, Budde WM, et al. Optical coherence of the optic nerve head: interindividual reproducibility. J Glaucoma. 2006;15:248-254.

31. Savini G, Espana EM, Acosta AC, et al. Agreement between optical coherence tomography and digital stereophotography in vertical cup-to-disc ratio measurement. Graefes Arch Clin Exp Ophthalmol. 2009;247:377-383.

32. Ramos Cdo V, Bellusci C, Savini G, et al. Association of optic disc size with development and prognosis of Leber's hereditary optic neuropathy. Invest Ophthalmol Vis Sci. 2009;50:1666-1674.

33. Dong J, Chihara E. Slope analysis of the optic disc in eyes with ocular hyper tension and early normal tension glaucoma by confocal scanning laser ophthalmoscope. $\mathrm{Br} J$ Ophthalmol. 2001;85:56-62.

34. Cullinane AB, Waldock A, Diamond JP, et al. Optic disc cup slope and visual field indices in normal, ocular hypertensive and early glaucomatous eyes. Br J Ophthalmol. 2002;86:555-559.

35. Hodapp E, Parrish RK II, Anderson DR. Clinical Decisions in Glaucoma. St Louis MO: Mosby; 1993:52-61. 